\title{
Progress on Perturbative Matching Calculations for the Charm Quark Mass using the HISQ Action
}

\author{
Emel Dalgic ${ }^{* a b}$, Kit Wong $^{c}$, Christine Davies ${ }^{c}$, Eduardo Follana ${ }^{d}$,Alistair Hart ${ }^{e}$,Ron \\ Horgan $^{f}$, Peter Lepage $^{g}$, Quentin Mason ${ }^{f}$, Junko Shigemitsu ${ }^{d}$, Howard Trottier $^{a}$ and \\ Jackson $\mathbf{W u}^{b}$ \\ ${ }^{a}$ Simon Fraser University, Burnaby BC, Canada V5A 1S6 \\ ${ }^{b}$ TRIUMF, Vancouver BC, Canada V6T $2 A 3$ \\ ${ }^{c}$ University of Glasgow, Glasgow, UK G12 $8 Q Q$ \\ ${ }^{d}$ The Ohio State University, Columbus OH, USA 43210 \\ ${ }^{e}$ University of Edinburgh, Edinburgh, UK EH9 3JZ \\ ${ }^{f}$ University of Cambridge, Cambridge, UK CB3 OHE \\ ${ }^{g}$ Cornell University, Ithaca NY, USA 14853 \\ E-mail: emel@triumf.ca,k.wong@physics.gla.ac.uk, \\ c.davies@physics.gla.ac.uk, e.follana@physics.gla.ac.uk, \\ a.hart@ed.ac.uk, R.R.Horgan@damtp.cam.ac.uk , \\ gpl@mail.lns.cornell.edu, quentin-mason@cornell.edu, \\ shige@pacific.mps.ohio-state.edu, trottier@sfu.ca, jwu@triumf.ca
}

\begin{abstract}
The highly-improved staggered quark (HISQ) action is the most accurate discretization scheme to date for the charm quark. Here we report on the progress of perturbative matching for the quark mass using the HISQ action. The matching is done through $O\left(\alpha_{s}^{2}\right)$ using a combination of Monte Carlo simulations at weak coupling and diagrammatic perturbation theory. When combined with on-going simulation efforts using the HISQ action, a determination of the charm quark mass to a few percent accuracy can be achieved. Of particular interest will be a comparison with the recent sum rule determination of the charm mass due to Kühn et al. [1].
\end{abstract}

The XXV International Symposium on Lattice Field Theory

July 30-4 August 2007

Regensburg, Germany

\footnotetext{
${ }^{*}$ Speaker.
} 


\section{Introduction}

Quark masses are fundamental parameters that go in the standard model and it is important to determine them precisely to constrain models beyond the standard model and to use them as inputs for phenomenological calculations. As we do not find free quarks in nature, their masses cannot be directly measured. One needs to instead do a comparison between lattice and experiment to extract quark masses. Quarks interact via the strong force, therefore such a calculation would be nonperturbative. Lattice QCD methods are well suited for this problem. This work builds on recent developments in staggered quarks [2].

Previously, the light quark masses $m_{u, d}^{\overline{M S}}$ and $m_{s}^{\overline{M S}}$ have been determined by several collaborations. We list some $m_{s}^{\overline{M S}}$ determinations. The HPQCD collaboration used the AsqTad action with 3 dynamical quarks to determine $m_{s}^{\overline{M S}}=87 \pm 4 \pm 4 \mathrm{MeV}$ [3]. The CP-PACS and JLQCD collaborations used the Wilson action with 3 dynamical quarks to calculate $m_{s}^{\overline{M S}}=91.1_{6.2}^{14.6} \mathrm{MeV}$ [4] . The QCDSF-UKQCD collaborations used clover fermions with 2 flavors of sea quarks to get $m_{s}^{\overline{M S}}=110-130 \mathrm{MeV}$ [5]. The ALPHA collaboration used Wilson quarks with 2 dynamical quarks and obtained $m_{s}^{\overline{M S}}=97(22) \mathrm{MeV}$ [6]. The $S P Q c d R$ collaboration used Wilson Quarks with 2 dynamical flavors to get $m_{s}^{\overline{M S}}=101(8)\left(\begin{array}{c}25 \\ 0\end{array}\right) \mathrm{MeV}$ [7]. The Particle Data Group reports the value $m_{s}^{\overline{M S}}=95 \pm 25 \mathrm{MeV}[8]$.

Our goal is to also determine $m_{c}$ with the HISQ (Highly Improved Staggered) quark action to a few percent accuracy, which will be determined by taste-changing effects and discretization. The taste changing effects for the HISQ action are up to 3-4 times smaller than for the Asqtad action as detailed in [2], and a comparison of the two actions is shown in Fig. 1. It will be interesting to compare our results for $m_{c}$ with the sum rules calculation by Kühn et al. [1], where an error of $1 \%$ is quoted.

Our aim is to do a perturbative matching calculation and obtain the renormalization factors for $m_{c}$ in the $\overline{M S}$ scheme. Below we list the perturbative expansions of the pole mass in terms of the bare mass, its relation to the $\overline{M S}$ mass, and the relevant mass renormalization factors:

$$
\begin{aligned}
m^{\text {Pole }} & =m_{0}\left[1+\alpha_{\text {lat }}\left(A_{11} \log \left(m_{0} a\right)+A_{10}\right),\right. \\
& \left.+\alpha_{\text {lat }}^{2} \log ^{2}\left(m_{0} a\right)+A_{21} \log \left(m_{0} a\right)+A_{20}+\ldots\right], \\
m^{\overline{M S}}(\mu) & =m^{\text {Pole }}\left(1+Z_{1}\left(\frac{\mu}{m^{\text {Pole }}}\right) \frac{\alpha_{\overline{M S}}}{\pi}+Z_{2}\left(\frac{\mu}{m^{\text {Pole }}}\right) \frac{\alpha_{\overline{M S}}^{2}}{\pi^{2}}\right), \\
m^{\overline{M S}}(\mu) & =\frac{a m_{0}}{a} Z_{m}\left(\mu a, m_{0} a\right), \\
Z_{m}\left(\mu a, m_{0} a\right) & =1+Z_{m, 1}(\mu a) \alpha_{V}\left(q^{*}\right)+Z_{m, 2}(\mu a) \alpha_{V}^{2}+\ldots
\end{aligned}
$$

Note that the correct expansion parameter to use is the renormalized coupling $\alpha_{V}$, the perturbative series in $\alpha_{\text {lat }}$ is not well behaved.

\section{Diagrammatic Method}

One way to do the matching is to use perturbation theory and compute all the diagrams up to the order at which we work. Since matching corrects for the short distance effects brought about by 


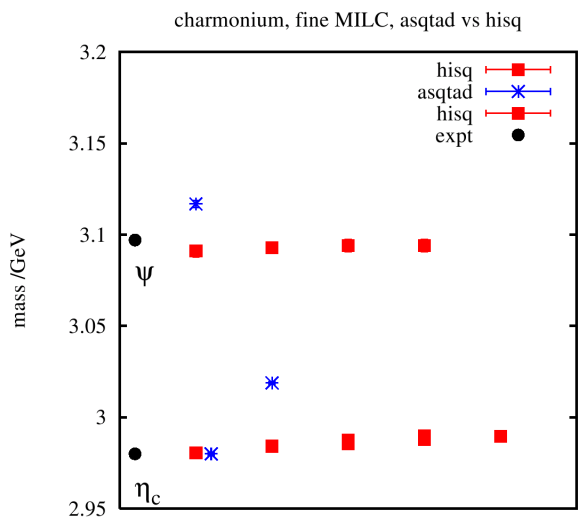

Figure 1: Comparison of results for the HISQ vs Asqtad actions [9]. The HISQ results correspond (from left to right) to 0-link, 1-link, 2-link and 3-link mesons. For Asqtad $\eta_{c}$ we show results for the 0-and 1-link pseudoscalars.

the finite lattice spacing, asymptotic freedom allows us to use perturbation theory. This approach is very involved, as it requires calculating many diagrams, and the Feynman rules for the HISQ action are extremely complicated. The relevant diagrams are shown in Fig. 2 .

\section{Another Method: Weak Coupling Monte Carlo}

An alternative to diagrammatic perturbation theory is to use Monte Carlo simulations at weak couplings, where the theory enters the perturbative phase. Simulations involving a particular operator, in this calculation the pole mass, are done at several values of the strong coupling, and the resulting data are then fitted to an expansion in $\alpha_{V}$ to yield the perturbative coefficients. The expectation value of an observable can be calculated on the lattice using

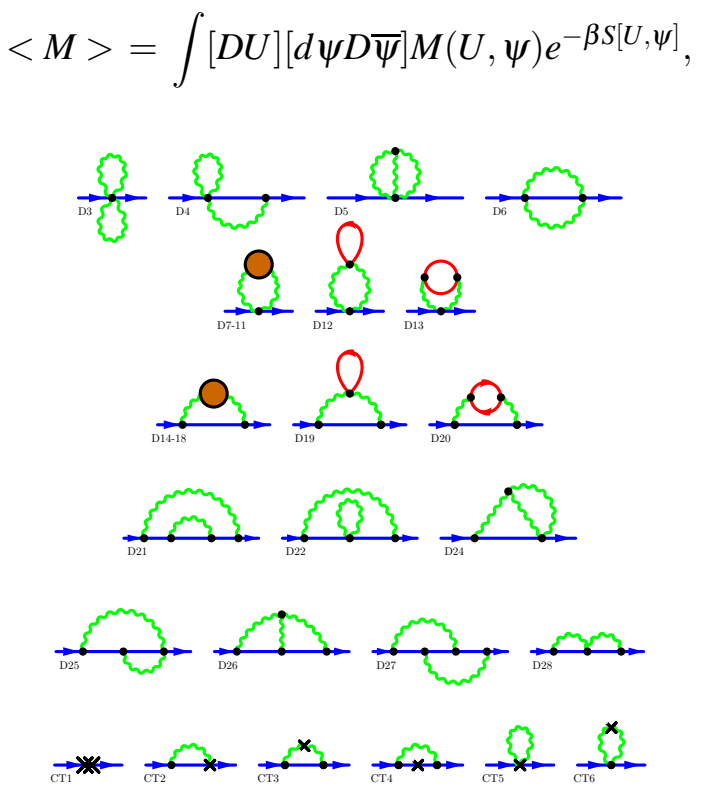

Figure 2: Relevant diagrams for the diagrammatic method. 


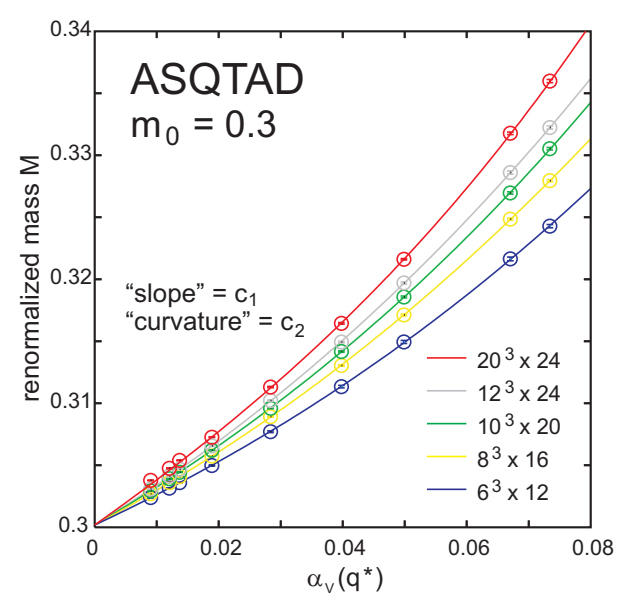

Figure 3: The renormalized mass $M$ measured at different couplings and lattice volumes for the ASQTAD action. The data points are fitted to an expansion in $\alpha_{V}\left(q^{*}\right)$ (solid lines). The "slopes" and "curvatures" are the first and second order coefficients respectively.

where $\beta=10 / g^{2}$. It is worth reiterating that in practice it is crucial to use the renormalized coupling instead of the bare lattice coupling $\alpha_{\text {lat }} \equiv g^{2} / 4 \pi$, for which perturbation theory is very poorly convergent. A good choice is $\alpha_{V}\left(q^{*}\right)$ defined by the static potential, along with an estimate of the optimal scale $q^{*}$ for the quantity of interest. The coupling is then converted back to $\alpha_{\text {lat }}$ using the known third order relation between $\alpha_{V}$ and $\alpha_{\text {lat }}$ [10].

As an example, Fig. 3 shows the renormalized mass $M$ measured at different couplings and lattice volumes for the ASQTAD action in the quenched approximation. The data points are fitted to an expansion of the form

$$
M=m_{\text {tree }}+c_{1} \alpha_{V}+c_{2}^{V} \alpha_{V}^{2}+\ldots,
$$

where $m_{\text {tree }}$ is the tree-level mass. The "slope" of the curve gives the first order coefficient $c_{1}$ (independent of the scheme) and the "curvature" is equal to $c_{2}^{V}$. One can use the $c_{1}$ value calculated from one loop perturbation theory to determine $c_{2}$ more accurately. Fig. 4a shows the infinite volume extrapolation of $c_{1}$. For comparison, results from diagrammatic perturbation theory [11], both at finite volume and in the infinite volume limit, are also plotted. Numerical values of $c_{2}$ can be calculated from Fig. 3 also by calculating the curvatures. To improve the accuracy, however, we re-fit the data with $c_{1}$ fixed to the analytic values at finite volume. Our results are shown in Fig. 4b. The overall agreement with our diagrammatic perturbation theory calculations is remarkable.

Fig. 5 shows the first order coefficients for the HISQ action, extrapolated to the infinite volume limit. For comparison, we also plot the same coefficients obtained using the diagrammatical method, and observe that the agreement is again outstanding. The 1-loop perturbative calculations at finite volume, which could be used to extract the second order coefficients from the data, are in progress.

Table 1 shows the perturbative coefficient $A_{20}$ calculated with the AsqTad action, neglecting sea quarks, compared to diagrammatic perturbation theory results. We find good agreement between the two sets of results.

We have demonstrated that perturbative coefficients for mass renormalization can be obtained with high accuracy from Monte Carlo simulations at weak couplings. This numerical method 

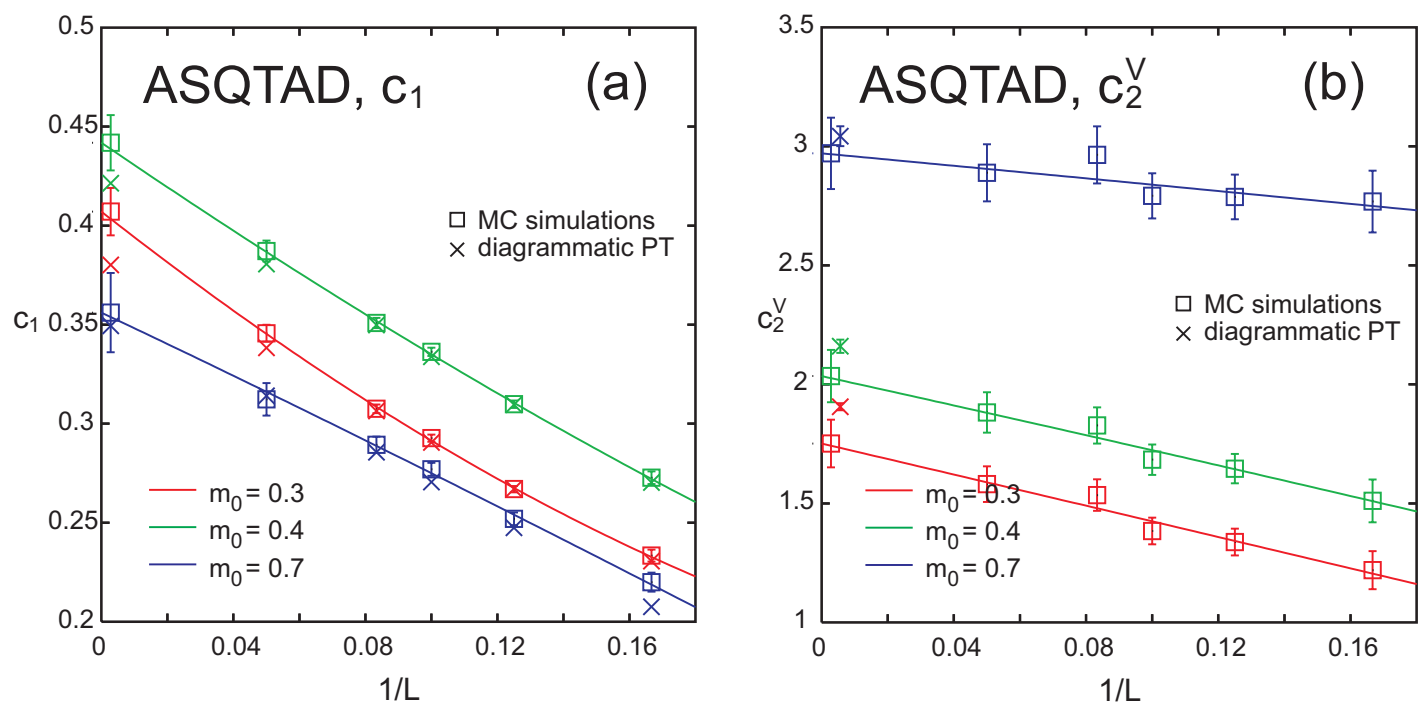

Figure 4: a) Infinite volume extrapolation of the first order coefficients. The error bars are invisible at this scale for the analytic results. b) Infinite volume extrapolation of the second order coefficients. Results are obtained by fixing $c_{1}$ to the analytic values at finite volume.

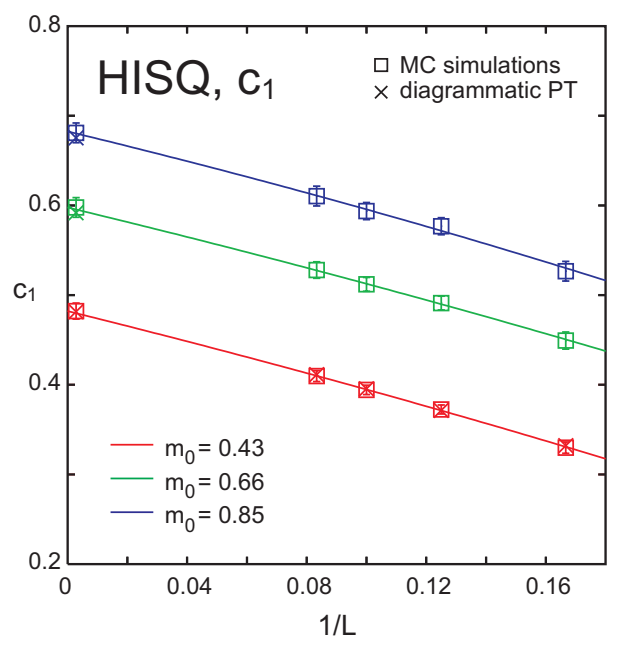

Figure 5: Infinite volume extrapolation of the first order coefficients for the HISQ action. The error bars are invisible at this scale for the analytic results.

\begin{tabular}{|c|c|c|}
\hline am & Diagrammatic PT & Weak Coupling MC \\
\hline 0.3 & $5.78(5)$ & $5.26(33)$ \\
0.4 & $5.61(7)$ & $5.30(28)$ \\
0.5 & $5.47(6)$ & $5.25(24)$ \\
0.6 & $5.23(6)$ & $5.18(22)$ \\
0.7 & $5.15(6)$ & $5.05(21)$ \\
\hline
\end{tabular}

Table 1: Asqtad quenched $A_{20}$, comparison between diagrammatic perturbation theory and high-beta Monte Carlo simulations. 


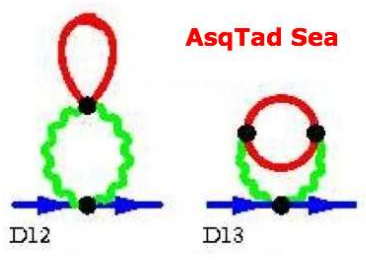

HISQ Valence

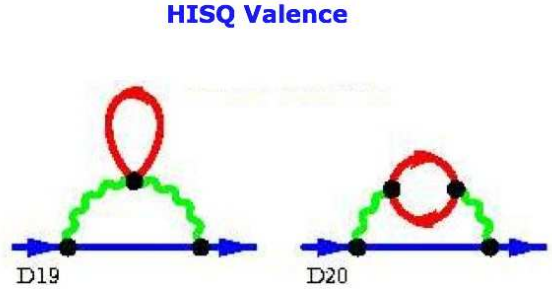

Figure 6: There are only 4 diagrams to compute in the fermionic part.

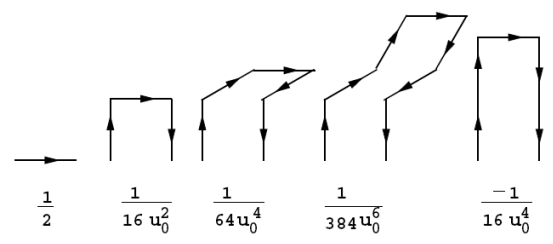

Figure 7: Fattening the link. This needs to be done twice, once with the thin link, and once more with the already fattened link.

provides a valuable alternative to diagrammatic perturbation theory.

\section{Strategy}

Our short term goal is to obtain the gluonic part of our calculation by the weak coupling Monte Carlo method, and get the fermionic part using diagrammatical perturbation theory. The diagrams needed are shown in Fig. 6.

In the long term, it would be desirable to do the entire calculation diagrammatically and also to compute the gluonic part using the Monte Carlo weak coupling approach in order to compare the two methods.

\section{Handling HISQ Perturbation Theory}

The HISQ action is obtained by fattening a simple link variable, reunitarizing the result, and fattening the resulting link again. Fattening is necessary for suppressing taste changing interactions, and fattening twice suppresses taste changing further. Reunitarization is necessary to suppress the unphysical tadpole diagrams which only exist in the lattice discretization and not the continuum limit. Fattening of a link can be visualised as in Fig. 7. Pieces of the action (in this case the links shown above) can be combined by doing Fourier convolutions, which means one assigns the gluons 


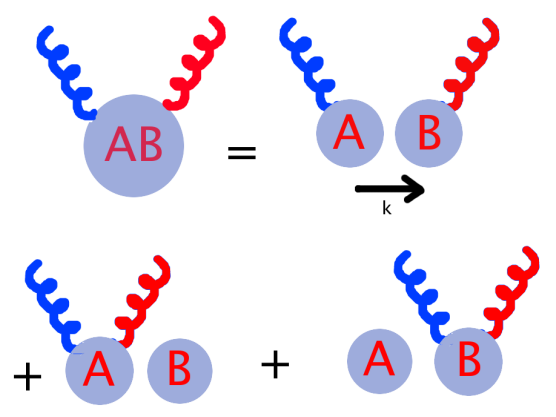

Figure 8: Convolution of two operators for two gluons.

to the pieces in all possible ways. Fig. 8 is a simple illustration with two operators and two gluons. Once we perform the convolutions and reunitarize, we obtain an improved link variable. We need to fatten this yet again. We therefore repeat the same process of convoluting, this time with the already fattened and reunitarized link, to obtain the full HISQ vertices.

\section{Conclusions}

Our project involves performing perturbative matching calculations to find $m_{c}$ through $O\left(\alpha_{s}^{2}\right)$ using the HISQ quark action. To achieve this, work is underway to do the fermionic part of the calculation diagrammatically, while the gluonic part is computed using the weak coupling Monte Carlo method. When both calculations are complete, the results will be combined to obtain perturbative coefficients for the $m_{C}$ mass renormalization. Our initial tests show that perturbative coefficients can be obtained accurately using the weak coupling Monte Carlo method. For the longer term goal of doing the entire calculation diagrammatically, the vertex functions necessary to achieve this aim have been prepared.

\section{References}

[1] J.H. Kühn et al., arXiv:hep-ph/0702103v1.

[2] E. Follana et al., Phys.Rev.D75, 054502 (2007).

[3] Q. Mason et al., Phys.Rev.D73,114501 (2006).

[4] T. Ishikawa et al., Nucl.Phys.Proc.Suppl.140,225 (2005).

[5] M. Göckeler et al., PoS LAT2005, 078 (2006).

[6] M. Della Morte et al., Nucl.Phys.B729,117 (2005).

[7] D. Becirevic et al., Nucl.Phys.B729,117 (2005).

[8] W. M. Yao et al., J. Phys. G33, 1 (2006).

[9] C. Davies et al., PoS LAT2006, 082 (2006).

[10] Q. Mason et al., HPQCD Collaboration, Phys. Rev. Lett. 95, 052002 (2005).

[11] Q. Mason et al., HPQCD Collaboration, Phys. Rev. D73, 114501 (2006). 